\title{
EXIT Chart Aided Design of Periodically Punctured Turbo Codes
}

\author{
Fulvio Babich, Alessandro Crismani \\ Dipartimento di Elettrotecnica, Elettronica ed Informatica \\ Università di Trieste, Via A. Valerio 10, 34127 Trieste, Italy \\ Tel: +39(040)558-7146; Fax: +39(040)558-3460 \\ e-mail: babich@units.it, alessandro.crismani@phd.units.it
}

\author{
Robert G. Maunder \\ Communications Research Group \\ School of Electronics and Computer Science \\ University of Southampton, Hampshire SO17 1BJ, UK \\ e-mail: rm@ecs.soton.ac.uk
}

\begin{abstract}
EXtrinsic Information Transfer (EXIT) charts have proved to be an effective tool for studying the convergence behaviour of iterative decoders. However, classical EXIT chart analysis fails to assess the performance of turbo codes in which the systematic bits are punctured periodically. In this letter, a novel 3-Dimensional (3D) EXIT chart technique is proposed for accurately modeling the convergence behaviour of turbo codes that are punctured following a periodic pattern. Finally, the novel 3D EXIT chart technique is validated for a particular periodic turbo code, which is shown to outperform the equivalent randomly punctured code.
\end{abstract}

Introduction: Turbo codes have been widely studied owing to their ability to closely approach Shannon's channel capacity. As shown in Figure 1, a turbo code encodes the bit sequence a using a parallel concatenation of convolutional encoders that are separated by an interleaver $\pi$. The resultant parity bit sequences $\mathbf{c}$ and $\mathbf{d}$ are multiplexed with the systematic bit sequence $\mathbf{a}$. Some bits are punctured and the rest are transmitted to the receiver, where zero values are used to represent the punctured bits in the Log-Likelihood Ratio (LLR) sequences $\tilde{\mathbf{a}}_{\mathrm{c}}, \tilde{\mathbf{c}}_{\mathrm{c}}$ and $\tilde{\mathbf{d}}_{\mathrm{c}}$. The Soft-In Soft-Out (SISO) decoders are operated iteratively, until convergence is achieved. This process may be accurately characterized by EXtrinsic Information Transfer (EXIT) charts [1], which provide a simple but effective tool for analysing and designing turbo codes. In particular, EXIT charts have been successfully employed to consider the effect of randomly puncturing the systematic bit sequence a produced by the turbo encoder [2]. However, in this letter we show that classical EXIT chart analysis fails to assess the performance of schemes in which the systematic bits a are punctured periodically. These schemes are of interest because parallel concatenations of periodically punctured convolutional codes have been studied and applied in Hybrid Automatic Repeat reQuest (HARQ) scenarios [3], [4]. In this letter, a novel 3-Dimensional (3D) EXIT chart technique is introduced to accurately model and design periodically punctured turbo codes, which are shown to outperform the equivalent randomly punctured code and to closely approach the performance of the corresponding turbo code in which only the parity bit sequences $\mathbf{c}$ and $\mathbf{d}$ are punctured.

Classical EXIT charts: A classical EXIT chart depicts the iterative Mutual Information (MI) exchange between a parallel concatenation of two SISO decoders. In particular, the a priori information provided to the upper decoder by the lower decoder is quantified by the MI $I\left(\tilde{\mathbf{a}}_{\mathrm{a}}\right)$ between the systematic bit sequence $\mathbf{a}$ and the a priori LLR sequence $\tilde{\mathbf{a}}_{\mathrm{a}}$ of Figure 1. In response, the upper decoder generates the extrinsic LLR sequence $\tilde{\mathbf{a}}_{\mathrm{e}}$, whose MI is given by the EXIT

This paper is a postprint of a paper submitted to and accepted for publication in Electronics Letters and is subject to Institution of Engineering and Technology Copyright. The copy of record is available at IET Digital Library.

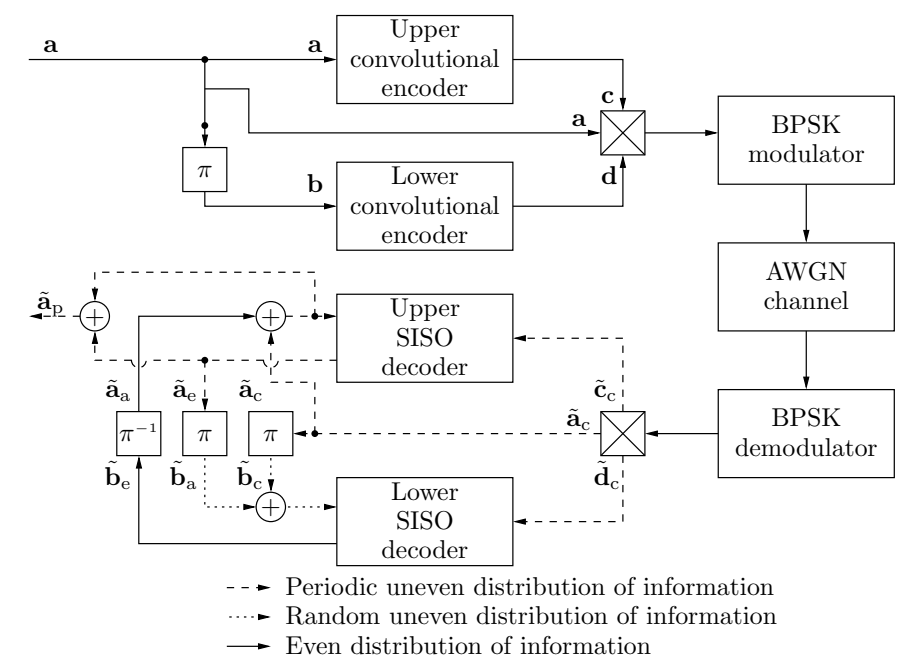

Fig. 1. Encoder and decoder for the considered turbo code.

function $I\left(\tilde{\mathbf{a}}_{\mathrm{e}}\right)=F_{\text {upper }}\left[I\left(\tilde{\mathbf{a}}_{\mathrm{a}}\right)\right]$. These extrinsic LLRs are provided to the lower decoder, which is characterized by the EXIT function $I\left(\tilde{\mathbf{a}}_{\mathrm{a}}\right)=F_{\text {lower }}\left[I\left(\tilde{\mathbf{a}}_{\mathrm{e}}\right)\right]$. The EXIT chart of Figure 2 plots the EXIT functions of a periodically punctured parallel concatenation of convolutional codes having the generator polynomial $(15)_{8}$ and the feedback polynomial (13) 8 . This was obtained for Binary Phase Shift Keying (BPSK) transmission over an Additive White Gaussian Noise (AWGN) channel having a Signal to Noise Ratio (SNR) of $1.55 \mathrm{~dB}$. The bit sequences $\mathbf{a}, \mathbf{c}$ and $\mathbf{d}$ are periodically punctured following the patterns a (1 11111000$), \mathbf{c}(00111000)$ and $\mathbf{d}(110000000)$ respectively, where a zero indicates that a bit is not transmitted. Figure 2 also provides the corresponding iterative decoding trajectory, which was obtained using a random interleaver $\pi$ having a length of $10^{5}$ bits. It can be seen that the EXIT function obtained for the lower decoder using classical EXIT chart analysis fails to predict the path of the iterative decoding trajectory. This is because the effect of puncturing bits in the systematic sequence $\mathbf{a}$ is to reduce the amount of extrinsic information for the neighbouring extrinsic LLRs in the sequence $\tilde{\mathbf{a}}_{\mathrm{e}}$. This effect is exacerbated if consecutive systematic bits in the sequence a are punctured, which happens consistently for periodic puncturing. Hence, when periodic puncturing is employed, the extrinsic LLRs in the sequence $\tilde{\mathbf{a}}_{\mathrm{e}}$ can be divided into two categories, those in the vicinity of punctured bits and those not in the vicinity of punctured bits. Since the former category has a higher MI than the latter one, there is an uneven distribution of information within $\tilde{\mathbf{a}}_{\mathrm{e}}$. However, classical EXIT chart analysis models the extrinsic LLR sequence $\tilde{\mathbf{a}}_{\mathrm{e}}$ using a single MI $I\left(\tilde{\mathbf{a}}_{\mathrm{e}}\right)$ and 


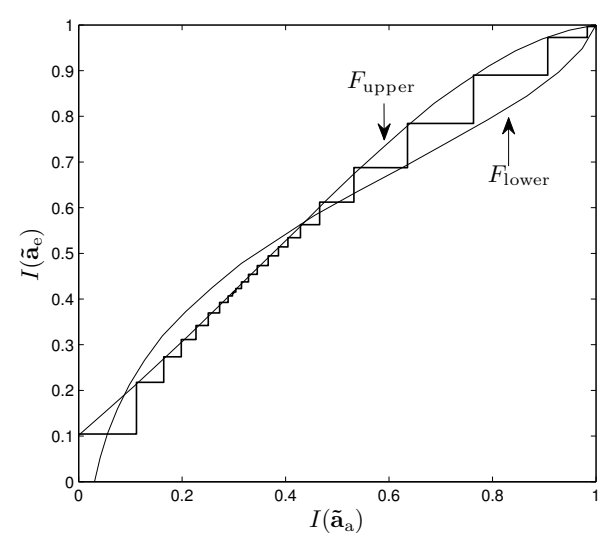

Fig. 2. Classical EXIT chart analysis of a periodically punctured turbo code, plotted jointly with a decoding trajectory.

hence an even distribution of information. Since the lower decoder is sensitive to the distribution of information in the extrinsic sequence $\tilde{\mathbf{a}}_{\mathrm{e}}$, the MI $I\left(\tilde{\mathbf{a}}_{\mathrm{a}}\right)$ of the a priori LLRs $\tilde{\mathbf{a}}_{\mathrm{a}}$ provided by it is not correctly predicted. Note also that classical EXIT chart analysis is able to correctly obtain the upper decoder's EXIT function. This is because the interleavers $\pi$ randomise the periodic uneven distribution of information within the LLR sequences $\tilde{\mathbf{a}}_{\mathrm{e}}$ and $\tilde{\mathbf{a}}_{\mathrm{c}}$ before they are provided to the lower decoder, as shown in Figure 2. As a result, the LLR sequence $\tilde{\mathbf{a}}_{\mathrm{a}}$ provided by the lower decoder has an even distribution of information, which is correctly modeled in classical EXIT chart analysis.

Novel 3D EXIT charts: Our novel approach divides the artificial LLR sequence $\tilde{\mathbf{a}}_{\mathrm{e}}$ into two categories. The first category $\tilde{\mathbf{a}}_{\mathrm{e}}^{\mathrm{p}}$ corresponds to the punctured bits of the sequence $\mathbf{a}$, while the second category $\tilde{a}_{\mathrm{e}}^{\mathrm{u}}$ corresponds to the unpunctured bits. The two categories of LLR $\tilde{\mathbf{a}}_{\mathrm{e}}^{\mathrm{p}}$ and $\tilde{\mathbf{a}}_{\mathrm{e}}^{\mathrm{u}}$ are generated separately using Gaussian distributions and having the distinct MIs $I\left(\tilde{\mathbf{a}}_{\mathrm{e}}^{\mathrm{u}}\right)$ and $I\left(\tilde{\mathbf{a}}_{\mathrm{e}}^{\mathrm{p}}\right)$, respectively. In this way, the EXIT function $I\left(\tilde{\mathbf{a}}_{\mathrm{a}}\right)=F_{\text {lower }}\left[I\left(\tilde{\mathbf{a}}_{\mathrm{e}}^{\mathrm{u}}\right), I\left(\tilde{\mathbf{a}}_{\mathrm{e}}^{\mathrm{p}}\right)\right]$ may be obtained. Additionally, the EXIT functions $I\left(\tilde{\mathbf{a}}_{\mathrm{e}}^{\mathrm{u}}\right)=F_{\mathrm{upper}}^{\mathrm{u}}\left[I\left(\tilde{\mathbf{a}}_{\mathrm{a}}\right)\right]$ and $I\left(\tilde{\mathbf{a}}_{\mathrm{e}}^{\mathrm{p}}\right)=F_{\text {upper }}^{\mathrm{p}}\left[I\left(\tilde{\mathbf{a}}_{\mathrm{a}}\right)\right]$ are evaluated separately. The novel 3D EXIT chart jointly plots the EXIT functions $I\left(\tilde{\mathbf{a}}_{\mathrm{a}}\right)=F_{\text {lower }}\left[I\left(\tilde{\mathbf{a}}_{\mathrm{e}}^{\mathrm{u}}\right), I\left(\tilde{\mathbf{a}}_{\mathrm{e}}^{\mathrm{p}}\right)\right]$, $I\left(\tilde{\mathbf{a}}_{\mathrm{e}}^{\mathrm{u}}\right)=F_{\text {upper }}^{\mathrm{u}}\left[I\left(\tilde{\mathbf{a}}_{\mathrm{a}}\right)\right]$ and $I\left(\tilde{\mathbf{a}}_{\mathrm{e}}^{\mathrm{p}}\right)=F_{\text {upper }}^{\mathrm{p}}\left[I\left(\tilde{\mathbf{a}}_{\mathrm{a}}\right)\right]$ together, as exemplified in Figure 3.

Results: Figure 3 shows the proposed 3D EXIT chart for a $R_{C}=$ $\frac{4}{5}$-rate turbo code, using the puncturing patterns a $(11111000)$,

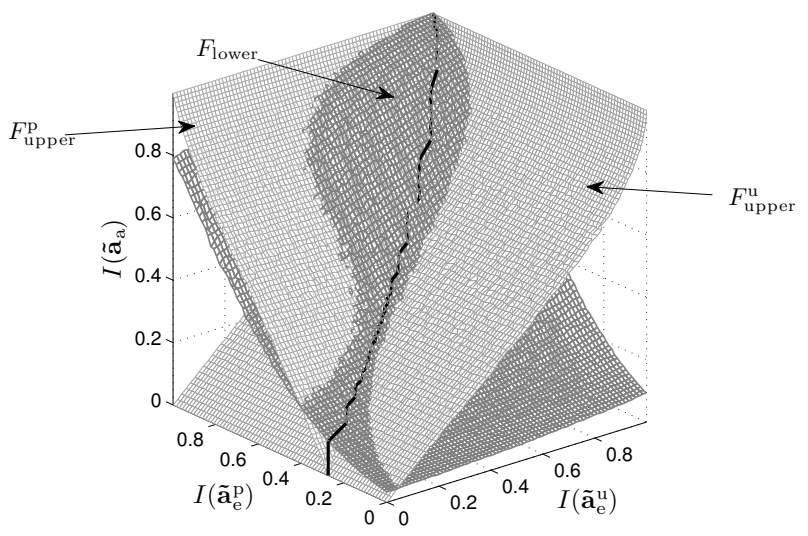

Fig. 3. Novel 3D EXIT chart analysis of a periodically punctured turbo code, plotted jointly with a decoding trajectory.

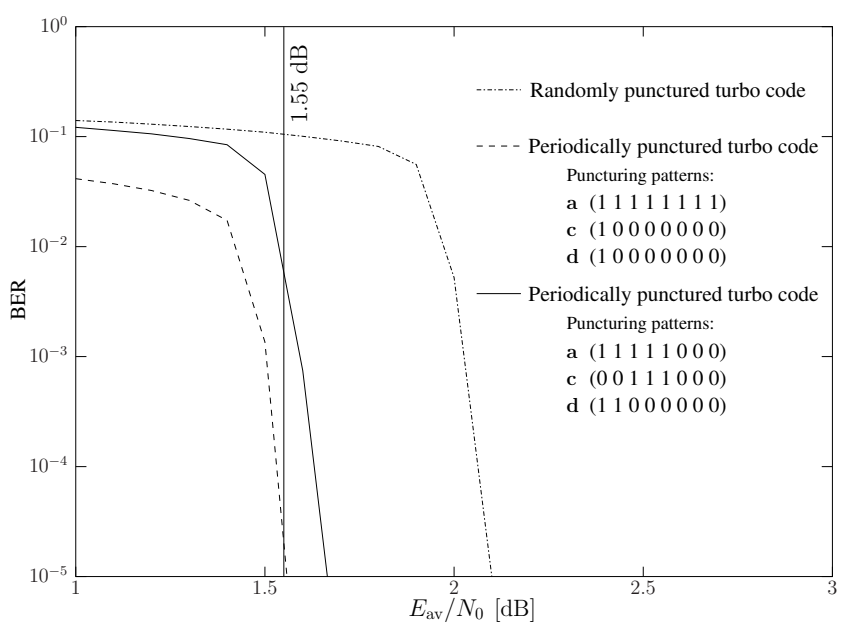

Fig. 4. BER versus $\frac{E_{a v}}{N_{0}}$ curves for various $R_{C}=\frac{4}{5}$-rate punctured turbo codes.

$\mathbf{c}(00111000)$ and $\mathbf{d}(11000000)$ for BPSK transmission on an AWGN channel having an SNR of $1.55 \mathrm{~dB}$. In contrast to the classical EXIT analysis of Figure 2, it can be seen that the proposed 3D EXIT chart closely predicts the decoding trajectory, which was obtained using a random interleaver $\pi$ having a length of $10^{5}$ bits. Our 3D EXIT chart technique was used as a design tool to select the above-mentioned puncturing patterns from the set of all possibilities for puncturing $\mathbf{a}, \mathbf{c}$ and $\mathbf{d}$ with fixed puncturing proportions. More explicitly, the chosen patterns are the ones offering an open tunnel at the lowest threshold SNR, namely $1.55 \mathrm{~dB}$, which is in agreement with the onset of the turbo cliff in the corresponding BER plot of Figure 4. Figure 4 also shows that our periodically punctured turbo code outperforms in terms of BER an equivalent randomly punctured turbo code, where $\frac{5}{8}$ of the systematic bits a and $\frac{3}{8}$ and $\frac{2}{8}$ of the parity bits $\mathbf{c}$ and $\mathbf{d}$ are preserved after puncturing. This fact may be explained by noting that a periodic puncturer provides more degrees of design freedom than random puncturing, facilitating improved EXIT chart matching. Furthermore, the performance of the code designed in this letter are close to that of the code where only parity bits $\mathbf{c}$ and $\mathbf{d}$ are punctured, despite the advantages in the turbo cliff region given by preserving the systematic bits after puncturing.

Conclusions: In this letter a novel 3D EXIT chart analysis was presented to successfully predict the iterative decoding convergence of turbo codes in which the systematic bits a are periodically punctured. The periodical punctured code designed with the aid of the novel 3D EXIT chart tool is shown to outperform the equivalent randomly punctured scheme and to closely approach the performance of turbo codes in which only the parity bits $\mathbf{c}$ and $\mathbf{d}$ are punctured.

\section{REFERENCES}

[1] S. ten Brink, "Convergence behavior of iteratively decoded parallel concatenated codes," Communications, IEEE Transactions on, vol. 49, no. 10 , pp. 1727-1737, 2001.

[2] F. Brannstrom and L. Rasmussen, "Multiple parallel concatenated codes with optimal puncturing and energy distribution," in IEEE International Conference on Communications, vol. 1, pp. 622-626, 2005.

[3] J. Hagenauer, "Rate-compatible punctured convolutional codes (RCPC codes) and their applications," Communications, IEEE Transactions on, vol. 36, no. 4, pp. 389-400, 1988.

[4] F. Babich and F. Vatta, "Robust, efficient and balanced (REB) ratecompatible puncturing schemes, for hybrid ARQ algorithms using turbo codes," in IEEE International Conference on Communications, vol. 3, pp. 1154-1159, June 2006. 